\title{
A COMPREHENSIVE APPROACH TO THE APPROXIMATE SOLUTION OF SINGULAR INTEGRAL EQUATIONS OVER THE ARC $(-1,1)$
}

\author{
DAVID ELLIOTT ${ }^{1}$
}

\begin{abstract}
Both a collocation and a Galerkin method are described for the approximate solution of the complete singular integral equation, with Cauchy principal value integral, defined on the arc $(-1,1)$. Algorithms are described for all values of the index and an analysis of the discrete equations is given for each case. The approach is based on polynomial approximation over the arc $(-1,1)$ and depends, in particular, upon the use of the Chebyshev polynomials of the first kind in the range space. The paper concludes with a convergence analysis which gives very satisfactory results. One surprising feature of the method is that except in the determination of the fundamental function, there is no need to evaluate any Cauchy principal value integrals. Furthermore, the method is not restricted to the particular cases of either constant or real coefficients and so is of wide applicability.
\end{abstract}

1. Introduction. In this paper singular integral equations of the form

$$
M \phi+K \phi=f,
$$

are considered where, if we let

$$
T \phi(t)=\frac{1}{\pi} \lambda \int_{-1}^{1} \frac{\phi(\tau) d \tau}{\tau-t}
$$

for $t \in(-1,1)$, the integral being taken as the Cauchy principal value, then

$$
M \phi(t)=a(t) \phi(t)+T(b \phi)(t)
$$

and

$$
K \phi(t)=\int_{-1}^{1} k(t, \tau) \phi(\tau) d \tau
$$

\footnotetext{
Research supported in part by the Australian Research Council (A.R.C.).
} 
Assume that the complex-valued functions $a, b, f$ and $k$ are given and we require $\phi$ or, in the present context, approximations to $\phi$. Then look for $\phi$ in the space of functions which is denoted by $\mathcal{H}\left(p_{1}, p_{2}\right)$ where $1 \leq p_{1}, p_{2} \leq \infty$. Such a function is Hölder continuous on the open interval $(-1,1)$ but is $p_{1}$-integrable at the end point -1 and $p_{2^{-}}$ integrable at the other end point +1 . The functions $a$ and $b$ are Hölder continuous on $[-1,1]$ and we shall put conditions on $k$ and $f$ as required below.

There has been much written in the past few decades on approximate solutions to (1.1)-(1.4); for two recent reviews see Golberg [3] and Venturino [10]. Generally $M$ has been defined as

$$
M \phi=a \phi+b T \phi
$$

but, as shown below, it is more convenient here to choose $M$ as defined by (1.3). Many approximate methods for solving singular integral equations have exploited the property that when $a$ and $b$ are real, the operator $M$ generates two sets of orthogonal polynomials. These polynomials have been used for both Galerkin and collocation methods to give approximate solutions. The theory is mathematically elegant although it has imposed upon it a further restriction that $b$ be a polynomial. The two sets of orthogonal polynomials turn out to be generalized Jacobi polynomials and their computation, in particular the calculation of their zeros and Christoffel numbers, has been a problem. In special cases, when the three term recurrence relation satisfied by these polynomials is known explicitly, the computation is straightforward. But when the recurrence relation is not known analytically it is much more difficult. In any case it has not been at all clear how to generalize these ideas to the case when $a$ and $b$ are complex-valued. Although the theory of singular integral equations allows $a$ and $b$ to be complex, these computational techniques appear to fail completely. Motivated by these considerations the author has been reviewing the usual approaches to the approximate solution of these equations. This paper is the outcome of these deliberations. One consequence has been to make a minor change, as noted above, to the definition of $M$. For completeness some of the theory of these equations is restated in $\S 2$, together with some other results which shall be needed later. In $\S 3$ we consider a Galerkin method based on polynomial approximation. First in $\S 3.1$ the dominant equation only is 
considered and then, in $\S 3.2$, the complete equation. All values of the index $\kappa$ are allowed, although we must consider separately the two cases of $\kappa \geq 1$ and $\kappa \leq 0$. In $\S 4$ a similar analysis for a collocation method is shown. Both the Galerkin and collocation methods are based on the use of Chebyshev polynomials of the first kind. As a consequence, the discretization of the dominant operator gives rise in all cases to matrices whose properties are readily determined. This considerably simplifies the computational problems. Another interesting aspect of these methods is that, other than when evaluating the fundamental function $Z$, there is no need to evaluate either exactly or approximately any Cauchy principal value integrals. We do, however, need to evaluate improper integrals and these may best be done by using appropriate adaptive quadrature schemes. In $\S 5$ we give an error analysis which embraces both methods described in $\S 3$ and $\S 4$. The upper bounds turn out to be very satisfactory and demonstrate convergence for many functions $f$ and $k$.

Because of its length we give no numerical results, but hope to repair this omission in a later paper.

2. Theory. Only a summary of results is given. For more information the reader is referred to the classic treatise of Muskhelishvili [7] (but see also Elliott $[2]$ ). For $t \in[-1,1]$ define

$$
r^{2}(t)=a^{2}(t)+b^{2}(t)
$$

and assume throughout that $r$ does not vanish on $[-1,1]$. A function $\theta$, which must be continuous on $[-1,1]$, is defined so that

$$
\exp \{ \pm i \pi \theta(t)\}=(a(t) \mp i b(t)) / r(t)
$$

where the upper and lower signs go together. The fundamental function $Z$ is defined on $(-1,1)$ by

$$
Z(t)=(1+t)^{n_{1}}(1-t)^{n_{2}} \exp \{\pi(T \theta)(t)\},
$$

where $n_{1}$ and $n_{2}$ are integers. These are determined by requiring that $Z \in \mathcal{H}\left(p_{1}, p_{2}\right)$, where $1 \leq p_{1}, p_{2} \leq \infty$, and $1 / Z \in \mathcal{H}\left(q_{1}, q_{2}\right)$ where $1 / p_{i}+1 / q_{i}=1, i=1,2$. If $\operatorname{Re} \theta(-1)$ is an integer then choose

$$
n_{1}=\operatorname{Re} \theta(-1)
$$


and similarly for $n_{2}$ if $\operatorname{Re} \theta(1)$ is an integer. However when neither is an integer look for $n_{1}$ and $n_{2}$ such that

$$
\left\{\operatorname{Re} \theta(-1)-1 / p_{1}\right\}<n_{1}<1+\left\{\operatorname{Re} \theta(-1)-1 / p_{1}\right\}
$$

and

$$
-\left\{\operatorname{Re} \theta(1)+1 / p_{2}\right\}<n_{2}<1-\left\{\operatorname{Re} \theta(1)+1 / p_{2}\right\} .
$$

The index $\kappa\left(p_{1}, p_{2}\right)$ of $M$ in the space $\mathcal{H}\left(p_{1}, p_{2}\right)$ is defined by

$$
\kappa\left(p_{1}, p_{2}\right)=-\left(n_{1}+n_{2}\right) \text {. }
$$

(Note that in the above analysis Muskhelishvili generally chooses $p_{1}=$ $\left.p_{2}=1\right)$. Consider now the deleted complex plane; that is the complex plane $\mathbf{C}$ with the interval $[-1,1]$ deleted. The canonical function $X(z)$ for $z \in \mathbf{C} \backslash[-1,1]$ is defined by

$$
X(z)=(1+z)^{n_{1}}(1-z)^{n_{2}} \exp \left\{\int_{-1}^{1} \frac{\theta(\tau) d \tau}{(\tau-z)}\right\} .
$$

From the Sokhotski-Plemelj formulae we find that for $t \in(-1,1)$

$$
X^{ \pm}(t)=\exp \{ \pm i \pi \theta(t)\} Z(t) \text {. }
$$

Now consider the solution of the dominant equation $M \phi=f$. It can be shown that for $1 \leq p_{1}, p_{2}<\infty, M$ maps the space $\mathcal{H}\left(p_{1}, p_{2}\right)$ into itself, so that we first assume that $f$ is a given element out of $\mathcal{H}\left(p_{1}, p_{2}\right)$ for $1 \leq p_{1}, p_{2}<\infty$. The operator $\hat{M}^{I}$ is defined by

$$
\hat{M}^{I} f=(Z / r)\{(a f / r Z)-T(f b / r Z)\} \text {. }
$$

For $\kappa \geq 1$, the general solution of $M \phi=f$ is given by

$$
\phi=\hat{M}^{I} f+(Z / r) \mathbf{P}_{\kappa-1},
$$

where $\mathbf{P}_{\kappa-1}$ denotes an arbitrary polynomial of degree $\leq(\kappa-1)$. On the other hand, when $\kappa \leq 0$ the solution of $M \phi=f$, if it exists, is unique and is given by

$$
\phi=\hat{M}^{I} f .
$$


Existence is guaranteed if $f$ satisfies the $-\kappa$ consistency conditions

$$
\int_{-1}^{1} \frac{\tau^{i-1} b(\tau) f(\tau)}{Z(\tau) r(\tau)} d \tau=0, \quad i=1(1)(-\kappa)
$$

We should note that only when $\kappa=0$ is $\hat{M}^{I}$ the inverse of $M$ and in this case the solution of $M \phi=f$ exists and is unique for all $f \in \mathcal{H}\left(p_{1}, p_{2}\right)$.

Now we introduce the principal part of a function defined in the complex plane $\mathbf{C}$. Suppose that the Laurent expansion of a function $f$ about the point at infinity is given by

$$
f(z)=\sum_{j=-\infty}^{N} f_{j} z^{j}, \quad f_{N} \frac{1}{\tau} 0 .
$$

Then the prinicpal part of $f$ at any point $z_{0} \in \mathbf{C}$ is denoted and defined by

$$
\text { p.p. }\left(f ; z_{0}\right)=\sum_{j=0}^{N} f_{j} z_{0}^{j} .
$$

If $N \leq-1$ then p.p. $(f) \equiv 0$ and if $N \geq 0$ then p.p. $(f)$ is a polynomial of degree $N$. By the theory of residues it can be shown that

$$
\text { p.p. }\left(f ; z_{0}\right)=\frac{1}{2 \pi i} \int_{C_{R}} \frac{f(z) d z}{z-z_{0}},
$$

where all the singularities of $f$ in the finite plane and the point $z_{0}$ are contained within the circle $C_{R}$, center at the origin and of radius $R$.

From (2.6) and (2.7) it can be shown that $X(z)$ behaves like $z^{-\kappa}$ at infinity; trivially, $X^{-1}(z)$ behaves like $z^{\kappa}$. It is straightforward to show that if $P$ is any polynomial then, for $t \in(-1,1)$,

$$
\frac{a(t) Z(t) P(t)}{r(t)}+T\left(\frac{b Z P}{r}\right)(t)=\text { p.p. }(P X ; t)
$$

and

$$
\frac{a(t) P(t)}{r(t) Z(t)}-T\left(\frac{b P}{r Z}\right)(t)=\text { p.p. }\left(P X^{-1} ; t\right)
$$


These two equations are of fundamental importance when we consider approximate methods of solution of (1.1).

Returning to equations (2.9) and (2.10), it is convenient to define a new dependent variable $\psi$ where

$$
\psi=r \phi / Z \text { or } \phi=Z \psi / r .
$$

In many cases $\psi$ will be a continuous function on $[-1,1]$. For this reason it is better to consider approximations to $\psi$ rather than $\phi$ which has integrable singularities at the end points. To be more specific, $Z$ has been chosen so that it is in $\mathcal{H}\left(p_{1}, p_{2}\right)$. Let operators $A$ and $\hat{A}^{I}$ be defined by

$$
A \psi=M(Z \psi / r), \quad \hat{A}^{I} \psi=(r / Z) \hat{M}^{I} \psi
$$

It can be shown (see Appendix) that $\hat{A}^{I}$ maps Hölder continuous functions of order $\nu$ on $[-1,1]$ into Hölder continuous functions of order $\nu-1 / q$ on $[-1,1]$ where $q=\min \left(q_{1}, q_{2}\right)$. Furthermore, it turns out that $\hat{A}^{I}$ is a bounded linear operator on the Hölder space $H_{\nu}[-1,1]$ into $C[-1,1]$. In terms of the operators $A$ and $\hat{A}^{I}$ we can rewrite $(2.16)$ and (2.17) respectively as

$$
A P=\text { p.p. }(P X), \quad \hat{A}^{I} P=\text { p.p. }\left(P X^{-1}\right),
$$

for all polynomials $P$. Thus both $A$ and $\hat{A}^{I}$ map polynomials into polynomials and this will be exploited in our algorithms.

From the definitions of $A$ and $\hat{A}^{I}$ it can be shown that

$$
\begin{cases}\hat{A}^{I} A=I \quad \text { when } \kappa \leq 0, \\ A \hat{A}^{I}=I \quad \text { when } \kappa \geq 0,\end{cases}
$$

so that $\hat{A}^{I}$ is a left inverse of $A$ when $\kappa \leq 0$ and a right inverse when $\kappa \geq 0$. Only when $\kappa=0$ is $\hat{A}^{I}$ the inverse of $A$. When $\kappa \geq 1$, the null space of $A$ is of dimension $\kappa$ and

$$
\operatorname{ker}(A)=\operatorname{span}\left\{1, t, \ldots, t^{\kappa-1}\right\} .
$$

Finally we make some comments on the polynomials which will be used throughout the algorithms to be discussed. We approximate 
to both $f$ and $\psi$ by polynomials so that we are concerned with global approximations on $[-1,1]$. For the range space of $A$ we shall approximate by means of the Chebyshev polynomials of the first kind. These are denoted and defined by

$$
T_{n}(x)=\cos n \theta, \quad x=\cos \theta
$$

A description of their properties is given in the book by Rivlin [9??], to which the reader will be frequently referred. In the domain of $A$ we introduce polynomials $u_{n}$, as follows. For $n \geq \max (0, \kappa)$ define

$$
u_{n}(t)=\left(\hat{A}^{I} T_{n-\kappa}\right)(t)=\text { p.p. }\left(X^{-1} T_{n-\kappa} ; t\right),
$$

on using (2.20). From the first equation of (2.24) it follows that

$$
\left(A u_{n}\right)(t)=T_{n-\kappa}(t)+\sum_{\ell=1}^{-\kappa} \sigma_{\ell, n} T_{\ell-1}(t)
$$

where the constants $\sigma_{\ell, n}$ are to be determined. We observe the convention that an empty sum is zero. When $\kappa \geq 0$ the sum is empty, when $\kappa \leq-1$ we have essentially an element out of the null space of the operator $\hat{A}^{I}$. It is readily seen from $(2.20)$ that

$$
\operatorname{ker}\left(\hat{A}^{I}\right)=\operatorname{span}\left\{1, t, \ldots, t^{-\kappa-1}\right\}
$$

For a given value of $n$, to determine the $(-\kappa)$ constants $\sigma_{l, n}$ we have that since, by construction, the solution $u_{n}$ of (2.25) exists, then the right hand side must satisfy the $(-\kappa)$ consistency conditions $(2.12)$. Thus we find

$$
\sum_{\ell=1}^{-\kappa}\left(\int_{-1}^{1} \frac{\tau^{k-1} b T_{\ell-1}}{r Z} d \tau\right) \sigma_{\ell, n}=-\int_{-1}^{1} \frac{\tau^{k-1} b T_{n-\kappa}}{r Z} d \tau,
$$

for $k=1(1)(-\kappa)$. This gives $(-\kappa)$ equations for $(-\kappa)$ unknowns $\sigma_{\ell, n}$ which, in principle, may be solved.

From time to time we need to evaluate finite sums of the form

$$
s_{n}(t)=\sum_{k=\lambda}^{n} c_{k-1} u_{k-1}(t), \quad \lambda=\max (1,1+\kappa),
$$


for given values of $t \in[-1,1]$, the coefficients $c_{k-1}$ being assumed known. A convenient method for doing this is based on a minor adaptation of Clenshaw's algorithm [1??]. Now for $n \geq 1$

$$
T_{n+1}(t)-2 t T_{n}(t)+T_{n-1}(t)=0
$$

with $T_{0}(t)=1$ and $T_{1}(t)=1$. For $n \geq \lambda$ we have, from (2.24) and (2.15) that

$$
\begin{aligned}
u_{n+1}(t)-2 t u_{n}(t)+u_{n-1}(t) & =2 p \cdot p \cdot\left((z-t) X^{-1}(z) T_{n-\kappa}(z) ; t\right) \\
& =\frac{1}{\pi i} \int_{C_{R}} X^{-1}(z) T_{n-\kappa}(z) d z,
\end{aligned}
$$

where $R>1$, the right hand side being independent of $t$. On deforming the contour $C_{R}$ to either side of the interval $[-1,1]$ and recalling from (2.2) and (2.8) that

$$
\left(X^{-1}\right)^{+}-\left(X^{-1}\right)^{-}=2 i b / r Z,
$$

we find that for $n \geq \lambda$ the polynomials $u_{n}$ satisfy the inhomogeneous recurrence relation

$$
u_{n+1}(t)-2 t u_{n}(t)+u_{n-1}(t)=\alpha_{n}
$$

where

$$
\alpha_{n}=-\frac{2}{\pi} \int_{-1}^{1} \frac{b(\tau) T_{n-\kappa}(\tau)}{r(\tau) Z(\tau)} d \tau .
$$

Assume that the coefficients $\alpha_{n}$ can be evaluated either analytically or, more generally, by quadrature to any desired accuracy. In order to evaluate $s_{n}(t)$ for a given value of $t \in[-1,1]$ construct the sequence $\left\{b_{k}\right\}$ for $k=(n+2)(-1) \lambda$ such that

$$
\left\{\begin{array}{l}
b_{n+2}=b_{n+1}=0 \\
b_{k}-2 t b_{k+1}+b_{k+2}=c_{k-1} .
\end{array} \quad\right. \text { and }
$$

Then it is readily shown that 


$$
\begin{gathered}
s_{n}(t)=b_{\lambda} u_{\lambda-1}(t)+b_{\lambda+1}\left(u_{\lambda}(t)-2 t u_{\lambda-1}(t)\right) \\
+\sum_{k=\lambda+2}^{n} b_{k} \alpha_{k-2} .
\end{gathered}
$$

Note that in $(2.24)$ the integer $(n-\kappa)$ was introduced and this has appeared subsequently. It will appear increasingly when we discretize (1.1) in various ways. We shall henceforth write

$$
m=n-\kappa,
$$

and always assume that $m$ and $n$ are non-negative integers.

\section{A Galerkin method.}

3.1 The dominant equation. Consider now an approximate method for solving the dominant equation for $\psi$, that is

$$
A \psi=f
$$

The two cases of $\kappa \geq 1$ and $\kappa \leq 0$ will be considered separately. First suppose that $\kappa \geq 1$. Recalling (2.22) and also, from (2.24), that the polynomials $u_{n}$ are defined only for $n \geq \kappa$, we shall look for an approximate solution of (3.1.1) of the form

$$
\psi_{n}(t)=\sum_{j=1}^{\kappa} a_{j} T_{j-1}(t)+\sum_{j=\kappa+1}^{n} a_{j} u_{j-1}(t) .
$$

If the residual $r_{n}$ is defined by

$$
r_{n}=A \psi_{n}-f
$$

then on substituting (3.1.2) into (3.1.3) we find that

$$
r_{n}=\sum_{j=\kappa+1}^{n} a_{j} T_{j-1-\kappa}-f .
$$


For the Galerkin method, in order to determine the $m$ coefficients $\left\{a_{\kappa+1}, \ldots, a_{n}\right\}$ impose the $m$ conditions that

$$
\int_{-1}^{1}\left(1-\tau^{2}\right)^{-1 / 2} r_{n}(\tau) T_{i-1}(\tau) d \tau=0, \quad i=1(1) m
$$

If we define, for $i \in \mathbf{N}$,

$$
f_{i-1}=\left(1 / h_{i-1}\right) \int_{-1}^{1}\left(1-\tau^{2}\right)^{-1 / 2} f(\tau) T_{i-1}(\tau) d \tau
$$

where

$$
h_{i-1}=2 /\left(1+\delta_{i-1,0}\right) \pi
$$

then on substituting (3.1.4) into (3.1.5) we find that

$$
a_{i}=f_{i-\kappa-1}, \quad i=(\kappa+1)(1) n .
$$

Thus from (3.1.2) and (3.1.8) we have

$$
\psi_{n}(t)=\sum_{j=\kappa+1}^{n} f_{j-\kappa-1} u_{j-1}(t)+\sum_{j=1}^{\kappa} a_{j} T_{j-1}(t)
$$

where the coefficients $a_{1}, a_{2}, \ldots, a_{\kappa}$ remain to be determined. But we know that when $\kappa \geq 1, \psi$ is not determined uniquely unless a further $\kappa$ conditions are imposed upon it. Suppose that these same conditions are imposed upon $\psi_{n}$ in order to determine the coefficients $a_{j}, j=1(1) \kappa$.

So much for the case when $\kappa \geq 1$. Assume that $\kappa \leq 0$. To find an approximate solution of (3.1.1) now write

$$
\psi_{n}(t)=\sum_{j=1}^{n} a_{j} u_{j-1}(t) .
$$

Defining the residual as before by (3.1.3) then on substituting (3.1.10) into (3.1.3) and recalling (2.25) we find

$$
r_{n}(t)=\sum_{j=1}^{n} a_{j}\left\{T_{j-1-\kappa}(t)+\sum_{\ell=1}^{-\kappa} \sigma_{\ell, j-1} T_{\ell-1}(t)\right\}-f(t) .
$$


Again apply the $m$ conditions as given by (3.1.5). For $i=(-\kappa+1)(1) m$ we obtain

$$
\begin{gathered}
\sum_{j=1}^{n} a_{j} \int_{-1}^{1}\left(1-\tau^{2}\right)^{-1 / 2} T_{i-1}(\tau) T_{j-1-\kappa}(\tau) d \tau \\
=\int_{-1}^{1}\left(1-\tau^{2}\right)^{-1 / 2} T_{i-1}(\tau) f(\tau) d \tau
\end{gathered}
$$

from which is obtained

$$
a_{i}=f_{i-\kappa-1} \quad \text { for } \quad i=1(1) n .
$$

Thus we obtain all the unknown coefficients in (3.1.10) and hence $\psi_{n}$. What of the remaining equations? For $i=1(1)(-\kappa)$ we obtain from (3.1.5) and (3.1.11) that

$$
\begin{aligned}
& \sum_{j=1}^{n} a_{j} \sum_{\ell=1}^{-\kappa} \sigma_{\ell, j-1} \int_{-1}^{1}\left(1-\tau^{2}\right)^{-1 / 2} T_{i-1}(\tau) T_{\ell-1}(\tau) d \tau \\
& \quad=\int_{-1}^{1}\left(1-\tau^{2}\right)^{-1 / 2} T_{i-1}(\tau) f(\tau) d \tau
\end{aligned}
$$

from which the orthogonality of the Chebyshev polynomials gives

$$
\sum_{j=1}^{n} \sigma_{i, j-1} a_{j}=f_{i-1}, \quad i=1(1)(-\kappa) .
$$

Combining this equation with (3.1.13) the equations can be written in partitioned matrix form as

$$
\left[\frac{\sum_{-\kappa}}{\mathbf{I}_{n}}\right] \mathbf{x}_{n}=\mathbf{y}_{m}
$$

where

$$
\mathbf{x}_{n}^{T}=\left(a_{1}, a_{2}, \ldots, a_{n}\right)
$$

and

$$
\mathbf{y}_{m}^{T}=\left(f_{0}, f_{1}, \ldots, f_{m-1}\right)
$$


The matrix $\boldsymbol{\Sigma}_{-\kappa}$ is a $(-\kappa) \times n$ matrix such that

$$
\boldsymbol{\Sigma}_{-\kappa}=\left(\sigma_{i, j-1}\right), \quad i=1(1)(-\kappa), \quad j=1(1) n .
$$

From the theory of linear equations (3.1.16) possesses a solution if $\mathbf{y}_{m}$ is orthogonal to the null space of the matrix.

$$
\left[\boldsymbol{\Sigma}_{-\kappa}^{T} \mid \mathbf{I}_{n}\right]
$$

this being an $n \times m$ matrix. Suppose vectors $\mathbf{u}_{i}, i=1(1)(-\kappa)$ are defined by

$$
\mathbf{u}_{i}^{T}=\left(0, \ldots, 1, \ldots 0,-\sigma_{i, 0},-\sigma_{i, 1}, \ldots,-\sigma_{i, n-1}\right)
$$

where the 1 appears in the ith place. It is readily verified that the $(-\kappa)$ vectors (3.1.21) span the null space of the matrix (3.1.20). Consequently (3.1.16) possesses a solution if

$$
\mathbf{u}_{i}^{T} \mathbf{y}_{m}=0 \quad \text { for } \quad i=1(1)(-\kappa)
$$

In other words we require

$$
f_{i-1}=\sum_{j=-\kappa+1}^{m} f_{j-1} \sigma_{i, j-1+\kappa} \quad \text { for } i=1(1)(-\kappa)
$$

It is of interest to derive the consistency conditions for the original equation (3.1.1). Suppose

$$
f(t)=\sum_{j=1}^{\infty} f_{j-1} T_{j-1}(t)
$$

the series converging absolutely and uniformly for all $t \in[-1,1]$ then, from (2.12), we must have

$$
\sum_{j=1}^{\infty} f_{j-1}\left(\int_{-1}^{1} \frac{\tau^{i-1} b(\tau) T_{j-1}(\tau)}{r(\tau) Z(\tau)} d \tau\right)=0
$$


for $i=1(1)(-\kappa)$, if the dominant equation $A \psi=f$ is to possess a solution. On splitting the sum into two sums with $j=1(1)(-\kappa)$ and $j=(-\kappa+1)(1) \infty$ and using $(2.27)$ we find

$(3.1 .26)$

$$
\sum_{j=1}^{-\kappa}\left(\int_{-1}^{1} \frac{\tau^{i-1} b(\tau) T_{j-1}(\tau)}{r(\tau) Z(\tau)} d \tau\right)\left\{f_{j-1}-\sum_{\ell=-\kappa+1}^{\infty} f_{\ell-1} \sigma_{j, \ell-1+\kappa}\right\}=0
$$

In order that this be satisfied we shall impose the $(-\kappa)$ conditions

$$
f_{j-1}=\sum_{\ell=-\kappa+1}^{\infty} f_{\ell-1} \sigma_{j, \ell-1+\kappa}, \quad j=1(1)(-\kappa)
$$

On comparing this equation with $(3.1 .23)$ we see that the latter is a truncated version of (3.1.27) where essentially we put $f_{m+\ell}=0$ for $\ell \in \mathbf{N}$.

This completes the discussion of this particular Galerkin method for the dominant equation. This method is now extended to the complete equation.

3.2 The complete equation. In terms of $\psi$ the complete equation will be written as

$$
A \psi+\mathcal{K} \psi=f
$$

where

$$
\mathcal{K} \psi(t)=\int_{-1}^{1}(Z(\tau) / r(\tau)) k(t, \tau) \psi(\tau) d \tau
$$

To find approximate solutions to $(3.2 .1)$, proceed as before by considering separately the two cases of $\kappa \geq 1$ and $\kappa \leq 0$.

Suppose first that $\kappa \geq 1$. Choose an approximate solution $\psi_{n}$ as given by (3.1.2). The residual $r_{n}$ is now defined as

$$
r_{n}=A \psi_{n}+\mathcal{K} \psi_{n}-f
$$


On substituting (3.1.2) into (3.2.3) and using (2.25), noting that the sum is now empty, we obtain

$$
\begin{aligned}
r_{n}(t)= & \sum_{j=\kappa+1}^{n} a_{j} T_{j-1-\kappa}(t)+\sum_{j=1}^{\kappa} a_{j} \int_{-1}^{1}(Z(\tau) / r(\tau)) k(t, \tau) T_{j-1}(\tau) d \tau \\
(3.2 .4) & +\sum_{j=\kappa+1}^{n} a_{j} \int_{-1}^{1}(Z(\tau) / r(\tau)) k(t, \tau) u_{j-1}(\tau) d \tau-f(t) .
\end{aligned}
$$

Now impose the $m$ conditions, as before, that

$$
\int_{-1}^{1}\left(1-t^{2}\right)^{-1 / 2} r_{n}(t) T_{i-1}(t) d t=0, \quad i=1(1) m
$$

Writing

$$
c_{j-1}(\tau)=\left(1 / h_{j-1}\right) \int_{-1}^{1}\left(1-t^{2}\right)^{-1 / 2} k(t, \tau) T_{j-1}(t) d t
$$

then (3.2.4) and (3.2.5) together give a system of $m$ linear algebraic equations in $n$ unknowns which can be written as

$$
\left(\mathbf{A}_{n}^{G}+\mathbf{K}_{n}^{G}\right) \mathbf{x}_{n}=\mathbf{y}_{m}
$$

The vectors $\mathbf{x}_{n}$ and $\mathbf{y}_{m}$ are as defined by (3.1.17) and (3.1.18) respectively. The matrix $\mathbf{A}_{n}^{G}$ is an $m \times n$ matrix which can be written in partitioned form as

$$
\mathbf{A}_{n}^{G}=\left(\mathbf{O}_{\kappa} \mid \mathbf{I}_{m}\right)
$$

where $\mathbf{O}_{k}$ denotes the null matrix of order $m \times \kappa$ and $\mathbf{I}_{m}$ is the identity matrix of order $m$. The matrix $\mathbf{K}_{n}^{G}$ can also be written in partitioned form as

$$
\mathbf{K}_{n}^{G}=\left(\mathbf{K}_{n, 1}^{G} \mid \mathbf{K}_{n, 2}^{G}\right)
$$

where, if $\mathbf{K}_{n, 1}^{G}=\left(k_{i, j}^{(1)}\right)$ then

$$
k_{i, j}^{(1)}=\int_{-1}^{1}(Z(\tau) / r(\tau)) c_{i-1}(\tau) T_{j-1}(\tau) d \tau
$$


for $i=1(1) m$ and $j=1(1) \kappa$. Again, if $\mathbf{K}_{n, 2}^{G}=\left(k_{i, j}^{(2)}\right)$ then

$$
k_{i, j}^{(2)}=\int_{-1}^{1}(Z(\tau) / r(\tau)) c_{i-1}(\tau) u_{j-1}(\tau) d \tau
$$

for $i=1(1) m$ and $j=(\kappa+1)(1) n$.

Before proceeding to discuss the solution of (3.2.7) consider briefly the integrals appearing in (3.2.10) and (3.2.11). If it is assumed that the functions $c_{i-1}$ are well behaved then the integrals will in general be improper integrals due to the singularities of the fundamental function $Z$ at the end points of the interval of integration. In the case of the integrals for the elements $k_{i, j}^{(2)}$, the polynomials $u_{j-1}$, for a given value of $\tau$, may be evaluated from the recurrence relation (2.32) which itself requires the values of the improper integrals $\alpha_{n}$ as defined by (2.33). It is suggested that adaptive quadrature rules may be used to evaluate all these integrals to any prescribed accuracy. The evaluation of the integrals $k_{i, j}^{(1)}$ may be done similarly.

Returning now to $(3.2 .7)$ partition the vector $\mathbf{x}_{n}$ so that

$$
\mathbf{x}_{n}^{T}=\left(\mathbf{x}_{n}^{(1) T} \mid \mathbf{x}_{n}^{(2) T}\right)=\left(a_{1}, \ldots, a_{\kappa} \mid a_{\kappa+1}, \ldots, a_{n}\right)
$$

Then rewrite $(3.2 .7)$ as

$$
\left(\mathbf{O}_{\kappa} \mid \mathbf{I}_{m}\right)\left(\frac{\mathbf{x}_{n}^{(1)}}{\mathbf{x}_{n}^{(2)}}\right)+\left(\left(\mathbf{K}_{n, 1}^{G} \mid \mathbf{K}_{n, 2}^{G}\right)\left(\frac{\mathbf{x}_{n}^{(1)}}{\mathbf{x}_{n}^{(2)}}\right)=\mathbf{y}_{m}\right.
$$

so that on multiplication we obtain

$$
\left(\mathbf{I}_{m}+\mathbf{K}_{n .2}^{G}\right) \mathbf{x}_{n}^{(2)}=\mathbf{y}_{m}-\mathbf{K}_{n .1}^{G} \mathbf{x}_{n}^{(1)}
$$

Now as has been seen in the previous section, the elements of $\mathbf{x}_{n}^{(1)}$ are arbitrary and can only be determined when $\kappa$ additional conditions are given. As is shown later (see $\S 5$ ) for $n$ (or $m$ ) large enough $\left(\mathbf{I}_{m}+\mathbf{K}_{n .2}^{G}\right)^{I}$ exists and then (3.2.14) can be solved. 
Suppose now that $\kappa \leq 0$. With $\psi_{n}$ as defined by (3.1.10) and $r_{n}$ as defined by (3.2.3), if we again impose the $m$ conditions given by(3.2.5), and recalling (2.25), a system of $m$ linear algebraic equations is obtained in $n$ unknowns which is written as

$$
\left(\frac{\boldsymbol{\Sigma}_{-\kappa}}{\mathbf{I}_{n}}\right) \mathbf{x}_{n}+\left(\frac{\mathbf{K}_{n, 1}^{G}}{\mathbf{K}_{n, 2}^{G}}\right) \mathbf{x}_{n}=\mathbf{y}_{m} .
$$

The vectors $\mathbf{x}_{n}$ and $\mathbf{y}_{m}$ are as defined by (3.1.17) and (3.1.18) respectively. The matrix $\boldsymbol{\Sigma}_{-\kappa}$ is defined by (3.1.19) and $\mathbf{I}_{n}$ is the identity matrix of order $n$. If we write

$$
\mathbf{K}_{n}^{G}=\left(\frac{\mathbf{K}_{n, 1}^{G}}{\mathbf{K}_{n, 2}^{G}}\right)=\left(k_{i, j}\right)
$$

then we have

$$
k_{i, j}=\int_{-1}^{1}(Z(\tau) / r(\tau)) c_{i-1}(\tau) u_{j-1}(\tau) d \tau
$$

for $i=1(1) m, j=1(1) n$ where $c_{i-1}$ is defined in (3.2.6). To solve (3.2.15) observe that the last $n$ equations give

$$
\left(\mathbf{I}_{n}+\mathbf{K}_{n .2}^{G}\right) \mathbf{x}_{n}=\mathbf{y}_{m}^{(2)}
$$

where

$$
\mathbf{y}_{m}^{(2) T}=\left(f_{-\kappa}, f_{-\kappa+1}, \ldots, f_{m-1}\right) .
$$

For $n$ large enough $\left(\mathbf{I}_{n}+\mathbf{K}_{n .2}^{G}\right)^{I}$ exists; see $\S 5$. However, there still remain $-\kappa$ conditions to be satisfied arising from the first $(-\kappa)$ equations of (3.2.15). That is, we must have

$$
\boldsymbol{\Sigma}_{-\kappa} \mathbf{x}_{n}+\mathbf{K}_{n, 1}^{G} \mathbf{x}_{n}=\mathbf{y}_{m}^{(1)},
$$

say where

$$
\mathbf{y}_{m}^{(1) T}=\left(f_{0}, f_{1}, \ldots, f_{-\kappa-1}\right) .
$$

If we take the second term on the left of (3.2.20) to the right hand side and assume, for the moment, that it is known, then we can apply 
the same arguments as were used for the dominant equation in $§ 3.1$. Consequently define, for $i=1(1) m$,

$$
g_{i-1}=f_{i-1}-\sum_{j=1}^{n} k_{i, j} a_{j}
$$

then from $(3.1 .23)$ the following $(-\kappa)$ conditions must be satisfied

$$
g_{i-1}=\sum_{j=-\kappa+1}^{m} g_{j-1} \sigma_{i, j-1+\kappa} .
$$

These may be verified a posteriori, once $\mathbf{x}_{n}$ has been determined from (3.2.18). Note as before, that these consistency conditions are a discretised version of those for the complete equation which, in terms of the dependent variable $\psi$, are given by

$$
\int_{-1}^{1} \frac{\tau^{i-1} b(\tau)}{r(\tau) Z(\tau)}\{f(\tau)-(\mathcal{K} \psi)(\tau)\} d \tau=0 .
$$

for $i=1(1)(-\kappa)$.

This completes the discussion of the Galerkin method. As has been seen the structure of the matrix arising from the operator $A$ is particularly simple in this case. However, this simplicity is obtained at a cost of evaluating many integrals and, in particular, the double integrals which arise in the coefficients $k_{i, j}$ of the matrix $\mathbf{K}_{n}^{G}$ (see (3.2.6) and (3.2.17). Inevitably these integrals will have to be evaluated approximately by some quadrature rule. This could be avoided by replacing (3.2.5) by $m$ different conditions. In particular one might consider a discrete Galerkin method where (3.2.5) is replaced by

$$
\sum_{j=1}^{m} r_{n}\left(\xi_{j, m}\right) T_{i-1}\left(\xi_{j, m}\right)=0
$$

for $i=1(1) m$ where $\xi_{j, m}$ are the $m$ zeros of $T_{m}$. We shall not pursue this any further in this paper but will turn our attentions now to a collocation method.

4. A collocation method. The same pattern shall be followed as in the previous section for the Galerkin method. First we shall consider 
the dominant equation and then, in $\S 4.2$, the complete equation. In each case we distinguish between two cases but now we choose them to be $\kappa \geq 0$ and $\kappa \leq-1$. The initial developments for this method are the same as for the Galerkin method and we shall be referring frequently to equations from the preceding section.

4.1. The dominant equation. Suppose first that $\kappa \geq 0$. With $\psi_{n}$ and $r_{n}$ as defined by(3.1.2) and (3.1.3), respectively, we obtain (3.1.4). In order to determine the $m$ coefficients $\left\{a_{\kappa+1}, \ldots, a_{n}\right\}$ now impose the $m$ conditions

$$
r_{n}\left(\xi_{i, m}\right)=0, \quad i=1(1) m,
$$

where the points $\xi_{i, m}$ are the $m$ zeros of $T_{m}$, that is

$$
\xi_{i, m}=\cos [(2 i-1) \pi /(2 m)], \quad i=1(1) m .
$$

Consequently a system of $m$ equations in $m$ unknowns is obtained and written as

$$
\mathbf{A}_{m} \mathbf{x}_{m}=\mathbf{y}_{m}
$$

where

$$
\mathbf{x}_{m}^{T}=\left(a_{\kappa+1}, \ldots, a_{n}\right)
$$

and

$$
\mathbf{y}_{m}^{T}=\left(f\left(\xi_{1, m}\right), \ldots, f\left(\xi_{m, m}\right)\right) .
$$

If we write $\mathbf{A}_{m}=\left(a_{i, j}\right)$ then

$$
a_{i, j}=T_{j-1}\left(\xi_{i, m}\right) \quad \text { for } \quad i, j=1(1) m .
$$

From the orthogonality property of the Chebyshev polynomials with respect to summation over the zeros of $T_{m}$ i.e.

$$
\sum_{i=1}^{m} T_{j-1}\left(\xi_{i, m}\right) T_{k-1}\left(\xi_{i, m}\right)=(m / 2)\left(1+\delta_{1, j}\right) \delta_{j, k}
$$


for $j, k=1(1) m$ (see $[\mathbf{9}$, Ex. 1.5.26]) we can write down explicitly the inverse of $\mathbf{A}_{m}$. If $\mathbf{A}_{m}^{I}=\left(a_{i, j}^{I}\right)$ then

$$
a_{i, j}^{I}=(2 / m) T_{i-1}\left(\xi_{j, m}\right) /\left(1+\delta_{i, 1}\right) \text { for } i, j=1(1) m .
$$

Consequently from (4.1.3) $\mathbf{x}_{m}=\mathbf{A}_{m}^{I} \mathbf{y}_{m}$ from which is obtained

$$
a_{i}=\left(2 / m\left(1+\delta_{i, 1}\right)\right) \sum_{j=1}^{m} T_{i-\kappa-1}\left(\xi_{j, m}\right) f\left(\xi_{j, m}\right)
$$

for $i=(\kappa+1)(1) n$. The remaining $\kappa$ coefficients $\left\{a_{1}, a_{2}, \ldots, a_{\kappa}\right\}$ must be determined from $\kappa$ additional conditions on $\psi_{n}$ which are assumed to be the same as those given for $\psi$.

When $\kappa \leq-1$ it is assumed that $\psi_{n}$ is given by (3.1.10) so that $r_{n}$ is given by (3.1.11). On imposing the $m$ conditions (4.1.1) we obtain a system of linear algebraic equations which is written as

$$
\left(\mathbf{A}_{m,-\kappa}+\mathbf{B}_{m} \boldsymbol{\Sigma}_{-\kappa}\right) \mathbf{x}_{n}=\mathbf{y}_{m},
$$

where

$$
\mathbf{x}_{n}^{T}=\left(a_{1}, a_{2}, \ldots, a_{n}\right)
$$

and $\mathbf{y}_{m}$ is defined in (4.1.5). If $\mathbf{A}_{m,-\kappa}=\left(a_{i, j}\right)$ then this is an $m \times n$ matrix where

$$
a_{i, j}=T_{j-1-\kappa}\left(\xi_{i, m}\right), \quad i=1(1) m, j=1(1) n .
$$

Again, $\mathbf{B}_{m}=\left(b_{i, j}\right)$ is an $m \times(-\kappa)$ matrix where

$$
b_{i, j}=T_{j-1}\left(\xi_{i . m}\right), \quad i=1(1) m, \quad j=1(1)(-\kappa) .
$$

Finally, the matrix $\boldsymbol{\Sigma}_{-\kappa}$ is a $(-\kappa) \times n$ matrix which has previously been defined in (3.1.19).

In order to solve (4.1.10) we first write it as

$$
\mathbf{A}_{m,-\kappa} \mathbf{x}_{n}=\mathbf{y}_{m}-\mathbf{B}_{m} \boldsymbol{\Sigma}_{-\kappa} \mathbf{x}_{n}
$$

and assume initially that the right hand side is known. In order that $\mathbf{x}_{n}$ exists, the right hand side must be orthogonal to the null space of 
$\mathbf{A}_{m,-\kappa}^{T}$. Again, from the orthogonality of the Chebyshev polynomials with respect to summation (see (4.1.7)), we can verify that there are $(-\kappa)$ linearly independent vectors $\mathbf{v}_{j}$ such that $\mathbf{A}_{m,-\kappa}^{T} \mathbf{v}_{j}=\mathbf{0}$ given by

$$
\mathbf{v}_{j}^{T}=\left(T_{j-1}\left(\xi_{1, m}\right), \ldots, T_{j-1}\left(\xi_{m, m}\right)\right)
$$

for $j=1(1)(-\kappa)$. Again from (4.1.7) it is shown that

$$
(2 / m) \mathbf{A}_{m,-\kappa}^{T} \mathbf{A}_{m,-\kappa}=\mathbf{I}_{n}
$$

and

$$
\mathbf{A}_{m,-\kappa}^{T} \mathbf{B}_{m}=\mathbf{0} .
$$

Consequently from (4.1.14)

$$
\mathbf{x}_{n}=(2 / m) \mathbf{A}_{m,-\kappa}^{T} \mathbf{y}_{m}
$$

provided that the $(-\kappa)$ consistency conditions on $\mathbf{y}_{m}$ given by

$$
\mathbf{v}_{j}^{T}\left(\mathbf{y}_{m}-(2 / m) \mathbf{B}_{m} \boldsymbol{\Sigma}_{-\kappa} \mathbf{A}_{m,-\kappa}^{T} \mathbf{y}_{m}\right)=0
$$

for $j=1(1)(-\kappa)$ are satisfied. Returning to (4.1.18) we see that provided $\mathbf{y}_{m}$ satisfies the $(-\kappa)$ conditions given by (4.1.19) then

$$
a_{i}=(2 / m) \sum_{j=1}^{m} T_{i-1-\kappa}\left(\xi_{j, m}\right) f\left(\xi_{j, m}\right)
$$

for $i=1(1) n$. This completes the discussion of the dominant equation so we now turn our attention to the collocation method as applied to the complete equation.

4.2. The complete equation.Consider separately the two cases of $\kappa \geq 0$ and $\kappa \leq-1$. When $\kappa \geq 0$ assume that $\psi_{n}$ is given by (3.1.2) then with $r_{n}$ as defined by (3.2.3) we find on imposing the $m$ conditions (4.1.1) that a system of $m$ equations in $n$ unknowns is obtained which we write as

$$
\left(\mathbf{A}_{m}^{C}+\mathbf{K}_{m}^{C}\right) \mathbf{x}_{n}=\mathbf{y}_{m}
$$


where now

$$
\mathbf{x}_{n}^{T}=\left(a_{1}, a_{2}, \ldots, a_{n}\right)
$$

and $\mathbf{y}_{m}$ is as defined in (4.1.5). The $m \times n$ matrix $\mathbf{A}_{m}^{C}$ can be written in partitioned form as

$$
\mathbf{A}_{m}^{C}=\left(\mathbf{O}_{\kappa} \mid \mathbf{A}_{m}\right)
$$

where $\mathbf{O}_{\kappa}$ denotes the $m \times \kappa$ null matrix and the $m \times m$ matrix $\mathbf{A}_{m}$ is defined in (4.1.6). The $m \times n$ matrix $\mathbf{K}_{m}^{C}$ is written in partitioned form as

$$
\mathbf{K}_{m}^{C}=\left(\mathbf{K}_{m, 1}^{C} \mid \mathbf{K}_{m, 2}^{C}\right)
$$

The matrix $\mathbf{K}_{m, 1}^{C}$ is an $m \times \kappa$ matrix where, if $\mathbf{K}_{m, 1}^{C}=\left(k_{i, j}^{(1)}\right)$, then

$$
k_{i, j}^{(1)}=\int_{-1}^{1}(Z(\tau) / r(\tau)) k\left(\xi_{i, m}, \tau\right) T_{j-1}(\tau) d \tau
$$

for $i=1(1) m$ and $j=1(1) \kappa$. The matrix $\mathbf{K}_{m, 2}^{C}$ is a square matrix of order $m$ where, if $\mathbf{K}_{m, 2}^{C}=\left(k_{i, j}^{(2)}\right)$, then

$$
k_{i, j}^{(2)}=\int_{-1}^{1}(Z(\tau) / r(\tau)) k\left(\xi_{i, m}, \tau\right) u_{j-1}(\tau) d \tau
$$

for $i=1(1) m$ and $j=(\kappa+1)(1) n$. On partitioning the vector $\mathbf{x}_{n}$ as in (3.2.12) we find on multiplying out the partitioned matrices that

$$
\left(\mathbf{A}_{m}+\mathbf{K}_{m, 2}^{C}\right) \mathbf{x}_{n}^{(2)}=\mathbf{y}_{m}-\mathbf{K}_{m, 1}^{C} \mathbf{x}_{n}^{(1)}
$$

Again note that the elements $a_{1}, a_{2}, \ldots, a_{\kappa}$ of $\mathbf{x}_{n}^{(1)}$ are arbitrary and can only be determined when additional $\kappa$ conditions are imposed upon $\psi_{n}$. Since there is an explicit representation for $\mathbf{A}_{m}$, see (4.1.8), we have from (4.2.7) that

$$
\left(\mathbf{I}_{m}+\mathbf{A}_{m}^{I} \mathbf{K}_{m, 2}^{C}\right) \mathbf{x}_{n}^{(2)}=\mathbf{A}_{m}^{I} \mathbf{y}_{m}-\mathbf{A}_{m}^{I} \mathbf{K}_{m, 1}^{C} \mathbf{x}_{n}^{(1)} .
$$


These equations may be solved for $\mathbf{x}_{n}^{(2)}$ in terms of the unknown elements of $\mathbf{x}_{n}^{(1)}$.

Finally, suppose that $\kappa \leq-1$. With $\psi_{n}$ as given by (3.1.10) and $r_{n}$ as defined by (3.2.3) then, on imposing the $m$ conditions (4.1.1) and using (2.25) we obtain a system of $m$ linear algebraic equations in $n$ unknowns given by

$$
\left(\mathbf{A}_{m,-\kappa}+\mathbf{B}_{m} \boldsymbol{\Sigma}_{-\kappa}+\mathbf{K}_{m}^{C}\right) \mathbf{x}_{n}=\mathbf{y}_{m} .
$$

The vectors $\mathbf{x}_{n}$ and $\mathbf{y}_{m}$ are as defined in (4.1.11) and (4.1.5), respectively. The matrices $\mathbf{A}_{m,-\kappa}, \mathbf{B}_{m}$ and $\boldsymbol{\Sigma}_{-\kappa}$ have been defined in (4.1.12), (4.1.13) and (3.1.19), respectively. The matrix $\mathbf{K}_{m}^{C}=\left(k_{i, j}\right)$ is an $m \times n$ matrix where

$$
k_{i, j}=\int_{-1}^{1}(Z(\tau) / r(\tau)) k\left(\xi_{i, m}, \tau\right) u_{j-1}(\tau) d \tau
$$

for $i=1(1) m$ and $j=1(1) n$. To solve (4.2.9) proceed as has been done for the dominant equation. Pre-multiplying (4.2.9) by $(2 / m) \mathbf{A}_{m,-\kappa}^{T}$ we obtain

$$
\left(\mathbf{I}_{n}+(2 / m) \mathbf{A}_{m,-\kappa}^{T} \mathbf{K}_{m}^{C}\right) \mathbf{x}_{n}=(2 / m) \mathbf{A}_{m,-\kappa}^{T} \mathbf{y}_{m}
$$

which gives us $n$ equations for $n$ unknowns. The solution $\mathbf{x}_{n}$ of (4.2.11) exists provided that the $(-\kappa)$ consistency conditions given by

$$
\mathbf{v}_{j}^{T}\left(\mathbf{y}_{m}-\left(\mathbf{B}_{m} \boldsymbol{\Sigma}_{-\kappa}+\mathbf{K}_{m}^{C}\right) \mathbf{x}_{n}\right)=0
$$

for $j=1(1)(-\kappa)$ are satisfied, where the vectors $\mathbf{v}_{j}$ are defined by (4.1.15). This condition may be checked a posteriori once the solution $\mathbf{x}_{n}$ has been obtained from (4.2.11).

This concludes the discussion of the discretization of the complete equation for the collocation method, for all values of the index $\kappa$. As in the Galerkin method, we evaluate only integrals which are improper. The slightly greater complexity of the algebraic equations in this case compared with those in the Galerkin method is offset by the need to evaluate only single integrals and not double integrals. Only in the evaluation of the fundamental function $Z$ do we need to evaluate a Cauchy principal value integral. It now remains to consider the 
convergence of these approximate methods and this will be done in the next section.

5. Convergence of the algorithms. For both the methods described in $\S 3$ and $\S 4$ the approximate solution $\psi_{n}$ satisfies an integral equation of the form

$$
A \psi_{n}+\mathcal{K}_{m} \psi_{n}=\boldsymbol{\Phi}_{m}
$$

In the Galerkin method of $\S 3$ both $\mathcal{K}_{m}$ and $\boldsymbol{\Phi}_{m}$ are obtained from truncated Chebyshev series expansions whereas for the collocation method of $\S 4$ they are obtained from Lagrange interpolation at the zeros of $T_{m}$. Compare the solution of (5.1) with that of

$$
A \psi+\mathcal{K} \psi=f
$$

When $\kappa>0$ assume that both $\psi$ and $\psi_{n}$ satisfy the same $\kappa$ additional conditions which have to be imposed for uniqueness. Define

$$
\varepsilon_{n}=\psi-\psi_{n},
$$

then from (5.1) and (5.2) we have, on subtraction, that

$$
A \varepsilon_{n}+\mathcal{K}_{m} \varepsilon_{n}=\left(f-\boldsymbol{\Phi}_{m}\right)+\left(\mathcal{K}-\mathcal{K}_{m}\right) \psi
$$

On regularizing this equation with the operator $\hat{A}^{I}$ we have that $\varepsilon_{n}$ satisfies

$$
\left(I+\hat{A}^{I} \mathcal{K}_{m}\right) \varepsilon_{n}=\hat{\mathbf{A}}^{i}\left(f-\boldsymbol{\Phi}_{m}\right)+\hat{A}^{I}\left(\mathcal{K}-\mathcal{K}_{m}\right) \psi
$$

Assume that $A, \mathcal{K}$ and $\mathcal{K}_{m}$ are linear operators on a normed space $X$ into a normed space $Y$, with $\mathcal{K}$ and $\mathcal{K}_{m}$ being compact. Furthermore, assume that $\hat{A}^{I}$ is a bounded linear operator on $Y$ into $X$. Consequently $\hat{A}^{I} \mathcal{K}_{m}$ will be compact so that (5.5) is a Fredholm integral equation. Later we shall choose appropriate spaces $X$ and $Y$. We assume that $\left(I+\hat{A}^{I} \mathcal{K}\right)^{I}$ exists and is bounded on $X$ into itself and we first consider the existence of $\left(I+\hat{A}^{I} \mathcal{K}_{m}\right)^{I}$. Now

$$
I+\hat{A}^{I} \mathcal{K}_{m}=\left(I+\hat{A}^{I} \mathcal{K}\right)\left\{I+\left(I+\hat{A}^{I} \mathcal{K}\right)^{I} \hat{A}^{I}\left(\mathcal{K}_{m}-\mathcal{K}\right)\right\}
$$


If we write the matrix in \{\} as $I+B_{m}$ and if $\left\|B_{m}\right\|<1$ for $m>m_{0}$ then it is well known that $\left(I+B_{m}\right)^{I}$ exists for $m>m_{0}$ and furthermore that $\left\|\left(I+B_{m}\right)^{I}\right\|<1 /\left(1-\left\|B_{m}\right\|\right)$. Consequently, if

$$
\left\|\left(I+\hat{A}^{I} \mathcal{K}\right)^{I} \hat{A}^{I}\left(\mathcal{K}_{m}-\mathcal{K}\right)\right\|<1 \text { for } m>m_{0}
$$

then $\left(I+\hat{A}^{I} \mathcal{K}_{m}\right)^{I}$ exists and there exists $c>0$, independent of $m$ such that

$$
\left\|\left(I+\hat{A}^{I} \mathcal{K}_{m}\right)^{I}\right\| \leq c \forall m>m_{0} .
$$

Now (5.7) will be satisfied if $\left\|\mathcal{K}_{m}-\mathcal{K}\right\| \rightarrow 0$ as $m \rightarrow \infty$. From (5.5) we have that

$$
\left\|\varepsilon_{n}\right\| \leq c\left\{\mid \hat{A}^{I}\left(f-\boldsymbol{\Phi}_{m}\right)\|+\| \hat{A}^{I}\left(\mathcal{K}-\mathcal{K}_{m}\right) \psi \|\right\},
$$

provided $m>m_{0}$.

Now consider appropriate spaces $X$ and $Y$. Choose $Y$ to be the Hölder space of order $\nu$. If $g \in Y$ then we define $\|g\|_{Y}$ by

$$
\|g\|_{Y}=\max \left\{\|g\|_{\infty}, \sup _{t_{1} \neq t_{2}} \frac{\left|g\left(t_{1}\right)-g\left(t_{2}\right)\right|}{\left|t_{1}-t_{2}\right|^{\nu}}\right\} .
$$

Here $\|g\|_{\infty}=\max _{-1 \leq t \leq 1}|g(t)|$ as usual, and in the supremum we assume that $t_{1}, t_{2}$ take all values in $[-1,1]$. For $X$ choose the space of continuous functions on $[-1,1]$ with uniform norm so that $X=$ $C[-1,1]$. It can be shown (Appendix, Theorem A2) that $\hat{A}^{I}$ is a bounded linear operator on $H_{\nu}[-1,1]$ into $C[-1,1]$. Furthermore, suppose that $f^{(r)} \in H_{\nu}[-1,1]$; that is, the $r$ th order derivative of $f$ is Hölder continuous of order $\nu$ on $[-1,1]$ where $r=0$ corresponds to the function itself. Recall that, originally, we required $\phi \in \mathcal{H}\left(p_{1}, p_{2}\right)$ and we choose $Z \in \mathcal{H}\left(p_{1}, p_{2}\right)$ and $1 / Z \in \mathcal{H}\left(q_{1}, q_{2}\right)$ where $1 / p_{i}+1 / q_{i}=1$ for $i=1,2$. Suppose $q=\min \left(q_{1}, q_{2}\right)$. Again it can be shown (see Appendix, Theorem A6) that if $f^{(r)} \in H_{\nu}[-1,1]$, where $1 / q<\nu \leq 1$, and if $F=\hat{A}^{I} f$, then $F^{(r)} \in H_{\nu-1 / q}[-1,1]$.

Now consider $f-\boldsymbol{\Phi}_{m}$. For both the Galerkin and collocation cases it can be shown that

$$
\left\|f-\boldsymbol{\Phi}_{m}\right\|_{\infty} \leq(m-1)^{-(r+\nu)}\left(c_{1}+c_{2} \log (m-1)\right) .
$$


In the former case, when $\boldsymbol{\Phi}_{m}$ is the truncated Chebyshev series, the result follows from Theorem 3.3 of [9] and the observation that if $f^{(r)} \in H_{\nu}[-1,1]$ then $E_{m-1}(f) \leq c(m-1)^{-(r+\nu)}$ (see, for example, [8, Theorem 1.5]). For the collocation method when $\boldsymbol{\Phi}_{m}$ is the Lagrange interpolation polynomial of degree $\leq(m-1)$ to $f$, the result follows from Theorems 1.1 and 1.2 of [9] together with the aforementioned result for $E_{m-1}(f)$.

Let us now obtain an estimate for $\left\|\hat{A}^{I}\left(f-\boldsymbol{\Phi}_{m}\right)\right\|_{\infty}$. If we write

$$
e_{m}=f-\boldsymbol{\Phi}_{m}
$$

then from (2.9) and (2.19)

$$
\hat{A}^{I} e_{m}=e_{m} \hat{A}^{I} 1-I_{m}(t)
$$

where

$$
I_{m}(t)=\frac{1}{\pi} \int_{-1}^{1} \frac{b(\tau)}{r(\tau) Z(\tau)}\left[\frac{e_{m}(\tau)-e_{m}(t)}{\tau-t}\right] d \tau
$$

Suppose $m$ is so large that $\left(t-1 / m^{2}, t+1 / m^{2}\right) \subset(-1,1)$. We shall write

$$
I_{m}(t)=I_{m}^{(1)}(t)+I_{m}^{(2)}(t)+I_{m}^{(3)}(t)
$$

where the interval of integration in (5.14) is broken up into the three sub-intervals $\left(-1, t-1 / m^{2}\right),\left(t-1 / m^{2}, t+1 / m^{2}\right)$ and $\left(t+1 / m^{2}, 1\right)$ respectively. From (5.11)

$\left|I_{m}^{(1)}(t)\right| \leq 2\left(c_{1}+c_{2} \log (m-1)\right)(m-1)^{-(r+\nu)} \int_{-1}^{t-1 / m^{2}}|b / r Z|(t-\tau)^{-1} d \tau$.

Now $|b / r Z| \in L_{q}$ where $q=\min \left(q_{1}, q_{2}\right)$ so that on using Hölder's inequality we find

$$
\left|I_{m}^{(1)}(t)\right| \leq\left(c_{1}+c_{2} \log (m-1)\right) m^{-(r+\nu-2 / q)} .
$$

(Note that constants $c, c_{1}, c_{2}$ do not necessarily take the same value from one equation to the next). A similar argument shows that the 
same upper bound holds for $\left|I_{m}^{(3)}(t)\right|$. It remains to consider $I_{m}^{(2)}(t)$. By Kalandiya's Lemma [5], we have

$$
\left|e_{m}(\tau)-e_{m}(t)\right| \leq\left(c_{1}+c_{2} \log (m-1)\right)(m-1)^{-(r+\nu-2 \mu)}|\tau-t|^{\mu}
$$

where $0<\mu<\nu / 2$. Substituting this into the integral for $I_{m}^{(2)}$ and using Hölder's inequality we find, provided $\mu>1 / q$, that

$$
\left|I_{m}^{(2)}(t)\right| \leq\left(c_{1}+c_{2} \log (m-1)\right)(m-1)^{-(r+\nu-2 / q)} .
$$

Combining (5.17) and (5.19) we have

$$
\left\|\hat{A}^{I}\left(f-\boldsymbol{\Phi}_{m}\right)\right\|_{\infty} \leq\left(c_{1}+c_{2} \log (m-1)\right) \cdot(m-1)^{-(r+\nu-2 / q)},
$$

provided that $f^{(r)} \in H_{\nu}[-1,1]$ where $2 / q<\nu \leq 1$ for $r=0,1,2, \ldots$.

If we assume that $\partial^{r} k / \partial t^{r}$ is uniformly Hölder continuous of order $\nu$ for all $\tau \in[-1,1]$ on $[-1,1]$ then a similar estimate for $\left\|\hat{A}^{I}\left(\mathcal{K}-\mathcal{K}_{m}\right) \psi\right\|_{\infty}$ holds in both cases. Thus, from (5.9), under the assumed conditions we have

$$
\left\|\varepsilon_{n}\right\|_{\infty} \leq\left(c_{1}+c_{2} \log (m-1)\right) \cdot(m-1)^{-(r+\nu-2 / q)} .
$$

This may be considered to be an excellent result. For $\psi^{(r)} \in H_{\nu-1 / q}[-1,1]$ and, as a consequence of Jackson's theorem [8], we have

$$
E_{n}(\psi) \leq c n^{-(r+\nu-1 / q)} .
$$

The rate of convergence of $\psi_{n}$ to $\psi$ as given by (5.21) is worse than the best possible result for polynomial approximation to $\psi$ by only the factor $n^{1 / q} \log n$. Again, as $p$ approaches $1,1 / q$ approaches zero and the factor approaches $\log n$.

Consider mean convergence which, as can be seen below, can be considered under more general conditions than those given above. For this analysis return to the original equations (1.1)-(1.4). Suppose we choose $p_{1}=p_{2}=p$ and we want $\phi \in \mathcal{H}(p, p), 1<p<\infty$ where we impose the $L_{p}$ norm onto this space. The approximate methods of $\S 3$ and $\S 4$ yield an approximation $\psi_{n}$ which gives us $\phi_{n}$ where $\phi_{n}=(Z / r) \psi_{n}$. Thus $\phi_{n}$ satisfies the singular integral equation

$$
M \phi_{n}+K_{m} \phi_{n}=\boldsymbol{\Phi}_{m}
$$


where $\boldsymbol{\Phi}_{m}$ denotes the approximation to $f$ and

$$
K_{m} \phi=\int_{-1}^{1} k_{m}(t, \tau) \phi(\tau) d \tau
$$

where $k_{m}$ is the similar approximation to $k$ considered as a function of the first variable $t$. Proceeding as before:

$$
\left(I+\hat{M}^{I} K_{m}\right)\left(\phi-\phi_{n}\right)=\hat{M}^{I}\left(f-\boldsymbol{\Phi}_{m}\right)+\hat{M}^{I}\left(K-K_{m}\right) \phi .
$$

Consider in more detail the operator $\hat{M}^{I}$, (see (2.9)). Recall that $a, b$ and $r$ are Hölder continuous on $[-1,1]$ and $r$ never vanishes there. Now $Z$ is such that on $(-1,1)$ we have

$$
|Z|=(1-t)^{\alpha}(1+t)^{\beta}|\boldsymbol{\Omega}|
$$

where $|\boldsymbol{\Omega}|$ does not vanish on $[-1,1]$ and $\alpha$ and $\beta$ satisfy

$$
-1 / p<\alpha, \beta<1 / q \text {. }
$$

By Minkowski's inequality if $f \in L_{p}$, then from (2.9) we have

$$
\left\|\hat{M}^{I} f\right\|_{p} \leq c\left\{\|\phi\|_{p}+\|Z T(b f / r Z)\|_{p}\right\} .
$$

By Khevedelidze's Theorem [6] we have, for $1<p<\infty$,

$$
\|Z T(b f / r Z)\|_{p} \leq c\|f\|_{p} .
$$

Consequently, $\hat{M}^{I}$ is a bounded linear operator on $\mathcal{H}(p, p)$ into itself. Arguing as we did at the commencement of this section, if $\lim _{m \rightarrow \infty}\left\|K-K_{m}\right\|_{p}=0$ then, for large enough $m,\left(I+\hat{M}^{I} K_{m}\right)^{I}$ will exist and be uniformly bounded on $\mathcal{H}(p, p)$ into itself. Thus we find from (5.25),

$$
\left\|\phi-\phi_{n}\right\|_{p} \leq c\left\{\left\|f-\boldsymbol{\Phi}_{m}\right\|_{p}+\left\|K-K_{m}\right\|_{p}\right\} .
$$

To exhibit convergence, consider each term on the right hand side separately. Consider the collocation method only of $\S 4$. As we have already observed in this case $\boldsymbol{\Phi}_{m}=L_{m-1}(f)$, the Lagrange interpolation polynomial of degree $\leq(m-1)$ to $f$ based on the zeros of $T_{m}$. A well 
known theorem of Erdös and Feldheim (see, for example, [9, p. 49]) shows that for all $p>0$ and for all $f \in C[-1,1]$

$$
\lim _{m \rightarrow \infty} \int_{-1}^{1}\left|f(\tau)-L_{m-1}(f ; \tau)\right|^{p}\left(1-\tau^{2}\right)^{-1 / 2} d \tau=0 .
$$

Since $1 \leq\left(1-\tau^{2}\right)^{-1 / 2}$ on $(-1,1)$ it follows that

$$
\lim _{m \rightarrow \infty}\left\|f-\boldsymbol{\Phi}_{m}\right\|_{p}=0 .
$$

whenever $f$ is continuous on $[-1,1]$ and certainly for all $p \in(1, \infty)$. For the second term of (5.30) an upper bound is given by

$$
\left\|K-K_{m}\right\|_{p} \leq\left[\int_{-1}^{1} d t\left\{\int_{-1}^{1}\left|k(t, \tau)-k_{m}(t, \tau)\right|^{q} d \tau\right\}^{p / q}\right]^{1 / p}
$$

see, for example, [4, Theorem 3.4.10]. Again, if $k$, considered as a function of $t$, is continuous on $[-1,1]$ then as above,

$$
\lim _{m \rightarrow \infty}\left\|K-K_{m}\right\|_{p}=0 .
$$

Under these conditions we have convergence in the $p$ norm of $\phi_{n}$ to $\phi$. It would appear that the conditions on $f$ and $k$ could be generalized so that they are piecewise continuous on $[-1,1]$.

For the Galerkin case a similar argument shows convergence in the 2norm when $f$ and $k$, considered as a function of $t$, are square integrable.

6. Conclusion. This analysis of global methods, based on polynomial approximation, for the approximate solution of singular integral equations has addressed all the problems raised in $\S 1$. The use of Chebyshev polynomials of the first kind in the range space has led to simple discretizations of the singular integral operator so that the structure of the solution of the discrete equations has been readily exhibited. The orthogonality property of the Chebyshev polynomials with respect to summation provided a key element of the analysis of the discrete equations in the collocation case. The analysis of convergence has also been straightforward in both cases and again has made good 
use of the key position that Chebyshev polynomials hold among all the orthogonal polynomials.

As remarked elsewhere, the algorithm depends more on the evaluation of improper integrals than of Cauchy principal value integrals. It is only in the evaluation of the fundamental function $Z$ that a Cauchy principal value integral needs to be evaluated either exactly or approximately.

No restriction is now placed on the Hölder continuous functions $a$ and $b$. These may be complex-valued. Furthermore, the analysis no longer restricts $b$ to being a polynomial which was required in other algorithms. The approximate methods described here now sit comfortably with the theory of singular integral equations, which required no such restrictions. Again note that the methods are valid no matter what the value of the index turns out to be.

Some further work remains to be done. In particular, note two things. The first is that we have not considered any numerical results; these must await another paper. The second is that we have not given any a posteriori estimates of error. These should follow quite readily from the analysis in $\S 5$ but do perhaps more naturally belong to an implementation paper.

One final point may be mentioned. The algorithm depended, among other things, on the fact that the operators $A$ and $\hat{A}^{I}$ mapped polynomials into polynomials. A similar analysis readily shows that they also map meromorphic functions into meromorphic functions. This raises the interesting possibility of approximating to $f$ (and $k$ ) by rational functions, but this also will have to await a further paper.

\section{Appendix}

In this appendix we shall prove two results, which were important in the convergence analysis of $\S 5$, concerning properties of the operator $\hat{A}^{I}$. From (2.9) and (2.19) we have for any $g \in Y$

$$
\hat{A^{I}} g=\frac{a g}{r Z}-T\left(\frac{b g}{r Z}\right) .
$$

If $\hat{A}^{I}: Y \rightarrow X$, then we shall choose $X$ to be the space $C[-1,1]$ of continuous functions with uniform norm and $Y$ to be the Hölder space $H_{\nu}[-1,1]$ of order $\nu$ 
where if $g \in H_{\nu}[-1,1]$ then

$$
\|g\|_{Y}=\max \left\{\|g\|_{\infty}, \sup _{t_{1} \neq t_{2}} \frac{\left|g\left(t_{1}\right)-g\left(t_{2}\right)\right|}{\left|t_{1}-t_{2}\right|^{\nu}}\right\}
$$

Here the supremum is taken over all distinct points $t_{1}, t_{2}$ of $[-1,1]$ and we shall assume initially that $0<\nu \leq 1$. Then the norm of $\hat{A}^{I}$ is defined by

$$
\left\|\hat{A}^{I}\right\|=\sup _{g \in H_{\nu}[-1,1]}\left\|\hat{A}^{I} g\right\|_{\infty}\|g\|_{Y}
$$

and we shall first show that $\hat{A}^{I}$ is a bounded linear operator on $Y$ into $X$. One further definition is required; if

$$
w=b / r Z
$$

then from the conditions imposed on $Z$, see (2.5), we have that $w \in \mathcal{H}\left(q_{1}, q_{2}\right)$.

Theorem A1. Given $w \in \mathcal{H}\left(q_{1}, q_{2}\right)$, where $1<q_{1}, q_{2}<\infty$, let $q=\min \left(q_{1}, q_{2}\right)$. Suppose $g \in H_{\nu}[-1,1]$ where $1 / q<\nu \leq 1$. If

$$
G(t)=\int_{-1}^{1} w(\tau)\left(\frac{g(\tau)-g(t)}{\tau-t}\right) d \tau, \quad t \in[-1,1]
$$

then $G \in H_{\nu-1 / q}[-1,1]$.

Proof . Choose $h>0$ and let $t_{0}$ and $t_{1}=t_{0}+h$ be points of $[-1,1]$. We must discuss 3 cases:

(i) if $\left[t_{0}-2 h, t_{0}+2 h\right] \subset(-1,1)$ then shall write $\ell=\left[t_{0}-2 h, t_{0}+2 h\right]$,

(ii) if $t_{1}=t_{0}+h=1$ then we shall write $\ell=\left[t_{0}-2 h, 1\right]$,

(iii) if $t_{0}=-1$ then we shall write $\ell=[-1,-1+2 h]$.

In all three cases $L=(-1,1) \backslash \ell$. Now

$$
\begin{aligned}
G\left(t_{1}\right)-G\left(t_{0}\right) & =\left[\int_{L}+\int_{\ell}\right]\left[w(\tau)\left\{\frac{g(\tau)-g\left(t_{1}\right)}{\tau-t_{1}}-\frac{g(\tau)-g\left(t_{0}\right)}{\tau-t_{0}}\right\}\right] d \tau \\
& =I_{1}+I_{2} .
\end{aligned}
$$

Since $g \in H_{\nu}[-1,1]$, if $A$ denotes the Hölder constant of $g$ then

$$
\begin{aligned}
\left|I_{2}\right| & \leq A \int_{\ell} w(\tau)\left\{\left|\tau-t_{1}\right|^{\nu-1}+\left|\tau-t_{0}\right|^{\nu-1}\right\} d \tau \\
& =J_{1}+J_{2} .
\end{aligned}
$$


Again consider these in reverse order. Now for case (i)

$$
J_{2}=A \int_{t_{0}-2 h}^{t_{0}+2 h} w(\tau)\left|\tau-t_{0}\right|^{\nu-1} d \tau
$$

Since $q=\min \left(q_{1}, q_{2}\right)$ then $w$ is $q$-integrable on $(-1,1)$ so that applying Hölder's inequality to $J_{2}$ gives

$$
J_{2} \leq c\left(\int_{t_{0}-2 h}^{t_{0}+2 h}\left|\tau-t_{0}\right|^{p(\nu-1)} d \tau\right)^{1 / p}
$$

where $1 / p+1 / q=1$. Evaluating this integral gives

$$
J_{2} \leq c h^{\nu-1 / q}, \text { since } \nu-1 / q>0 .
$$

Note that $c$ denotes a generic constant whose value may be different in different places. Since we can argue similarly for the integral $J_{1}$ we find

$$
\left|I_{2}\right| \leq \mathrm{ch}^{\nu-1 / q} .
$$

Return to the integral $I_{1}$; rewrite this as

$$
I_{1}=\left(g\left(t_{0}\right)-g\left(t_{1}\right)\right) \int_{L} \frac{w(\tau) d \tau}{\tau-t_{0}}+h \int_{L} \frac{w(\tau)\left(g(\tau)-g\left(t_{1}\right)\right)}{\left(\tau-t_{0}\right)\left(\tau-t_{1}\right)} d \tau,
$$

so that

$$
\left|I_{1}\right| \leq A h^{\nu} \cdot J_{3}+J_{4} .
$$

Again, considering these integrals in reverse order

$$
\begin{aligned}
\left|J_{4}\right| & \leq A h \int_{L} \frac{w(\tau)\left|\tau-t_{1}\right|^{\nu-1}}{\left|\tau-t_{0}\right|} d \tau \\
& =A h \int_{L} \frac{w(\tau) d \tau}{\left|\tau-t_{0}\right|^{2-\nu}\left|1-h /\left(\tau-t_{0}\right)\right|^{1-\nu}}
\end{aligned}
$$

on recalling that $t_{1}=t_{0}+h$. Now on $L$ we have, for case (i),

$$
\frac{2}{3} \leq \frac{1}{1-h /\left(\tau-t_{0}\right)} \leq 2
$$

so that

$$
\left|J_{4}\right| \leq \operatorname{ch}\left\{\int_{-1}^{t_{0}-2 h} \frac{w(\tau) d \tau}{\left(t_{0}-\tau\right)^{2-\nu}}+\int_{t_{0}+2 h}^{1} \frac{w(\tau) d \tau}{\left(\tau-t_{0}\right)^{2-\nu}}\right\}
$$


Applying Hölder's inequality to each of these integrals we find, as before, that

$$
\left|J_{4}\right| \leq \operatorname{ch}^{\nu-1 / q} .
$$

Finally we have, using Hölder's inequality, that

$$
\begin{aligned}
\left|A h^{\nu} J_{3}\right| \leq \operatorname{ch}^{\nu}\left\{\left(\int_{-1}^{t_{0}-2 h}\left(t_{0}-\tau\right)^{-p} d \tau\right)\right)^{1 / p} & \\
& \left.+\left(\int_{t_{0}+2 h}^{1}\left(\tau-t_{0}\right)^{-p} d \tau\right)^{1 / p}\right\} \\
\leq & \operatorname{ch}^{\nu-1 / q}
\end{aligned}
$$

after some algebra. Thus $\left|I_{1}\right| \leq c h^{\nu-1 / q}$ and combining this with the bound for $\left|I_{2}\right|$ gives

$$
\left|G\left(t_{1}\right)-G\left(t_{0}\right)\right| \leq c h^{\nu-1 / q} .
$$

This analysis applies for case (i). Although we shall not do so here it can be shown that the same bound also applies in cases (ii) and (iii) and this establishes the theorem.

We now show that $\hat{A}^{I}$ is a bounded linear operator on $H_{\nu}[-1,1]$ into $C[-1,1]$.

Theorem A2. If $w \in \mathcal{H}\left(q_{1}, q_{2}\right), 1<q_{1}, q_{2}<\infty$, then $\hat{A}^{I}$ maps $H_{\nu}[-1,1]$ into $H_{\nu-1 / q}[-1,1]$ where $q=\min \left(q_{1}, q_{2}\right)$ and $1 / q<\nu \leq 1$. Furthermore $\hat{A}^{I}$ is a bounded linear operator on $H_{\nu}[-1,1]$ into $C[-1,1]$.

Proof. The linearity of $\hat{A}^{I}$ is obvious from (A1). From (2.17) with $P \equiv 1$ we have

$$
a / r Z=\text { p.p. }\left(X^{-1}\right)+T(w) .
$$

Substituting this into (A1) gives

$$
\hat{A}^{I} g=g \text { p.p. }\left(X^{-1}\right)-\frac{1}{\pi} \int_{-1}^{1} w(\tau)\left(\frac{g(\tau)-g(t)}{\tau-t}\right) d \tau .
$$

Now p.p. $\left(X^{-1}\right)$ is a polynomial so that $g$ p.p. $\left(X^{-1}\right) \in H_{\nu}[-1,1]$ and therefore in $H_{\nu-1 / q}[-1,1]$. That the second term is in $H_{\nu-1 / q}[-1,1]$ follows immediately from Theorem A1. Hence $\hat{A}^{I}$ maps $H_{\nu}[-1,1]$ into $H_{\nu-1 / q}[-1,1]$ and therefore into $C[-1,1]$. From $(\mathrm{A} 7)$

$$
\left|\left(\hat{A}^{I} g\right)(t)\right| \leq c\left\{|g(t)|+\int_{-1}^{1} w(\tau)\left|\frac{g(\tau)-g(t)}{\tau-t}\right| d \tau\right\},
$$


where $c$ is a constant independent of both $g$ and $t$. From (A2) we have that

$$
\left\|\hat{A}^{I} g\right\|_{\infty} \leq c .\|g\|_{Y}
$$

where $c$ is independent of $g$. This establishes the boundedness of $\hat{A}^{I}$.

Returning to (A5) we want to show that if $g$ possesses a Hölder continuous derivative of order $r$ then $G$ also possesses a Hölder continuous derivative of order $r$. To do this we need to introduce some auxiliary functions.

DEFinition. Suppose that for some $r \geq 1, g^{(r)} \in H_{\nu}[-1,1]$ where $0<\nu \leq 1$. On $[-1,1]$ we define

$$
\left\{\begin{array}{l}
g_{0}(\tau, t)=g(\tau) \\
g_{k}(\tau, t)=k \int_{0}^{1} g^{(k)}(\eta t+(1-\eta) \tau) \eta^{k-1} d \eta
\end{array}\right.
$$

for $k=1(1) r$.

We now have the following lemma

LEMma A3. If $g^{(r)} \in H_{\nu}[-1,1]$ then

(i) $g_{k}(t, t)=g^{(k)}(t), \quad k=0(1) r$

(ii) $\frac{\partial g_{k}}{\partial t}=\frac{k}{k+1} g_{k+1}, \quad k=0(1)(r-1)$,

(iii) $\frac{g_{k}(\tau, t)-g_{k}(t, t)}{\tau-t}=\frac{1}{k+1} g_{k+1}(\tau, t), \quad k=0(1)(r-1)$,

(iv) $g_{r}(\tau, t) \in H_{\nu}[-1,1] \times H_{\nu}[-1,1]$.

Proof. The proofs of (i) - (iii) follow immediately from the definition. For the proof of (iv), if we assume that

$$
\left|g^{(r)}\left(t_{1}\right)-g^{(r)}\left(t_{2}\right)\right| \leq A_{r}\left|t_{1}-t_{2}\right|^{\nu}
$$

for any $t_{1}, t_{2} \in[-1,1]$ then for every $\tau_{1}, \tau_{2}, t_{1}, t_{2} \in[-1,1]$ we have that

$$
\left|g_{r}\left(\tau_{1}, t_{1}\right)-g_{r}\left(\tau_{2}, t_{2}\right)\right| \leq r A_{r} \int_{0}^{1} \eta^{r-1}\left|\eta\left(t_{1}-t_{2}\right)+(1-\eta)\left(\tau_{1}-\tau_{2}\right)\right|^{\nu} d \eta .
$$

Now for $\sigma_{1}, \sigma_{2} \geq 0$ and $0<\nu<1$ we have that $\left(\sigma_{1}+\sigma_{2}\right)^{\nu} \leq \sigma_{1}^{\nu}+\sigma_{2}^{\nu}$ so that

$$
\begin{aligned}
\left|g_{r}\left(\tau_{1}, t_{1}\right)-g_{r}\left(\tau_{2}, t_{2}\right)\right| \leq r A_{r}\left\{\left|t_{1}-t_{2}\right|^{\nu} \int_{0}^{1} \eta^{r-1+\nu} d \eta\right. \\
\left.+\left|\tau_{1}-\tau_{2}\right|^{\nu} \int_{0}^{1} \eta^{r-1}(1-\eta)^{\nu} d \eta\right\}
\end{aligned}
$$


from which the result follows.

With these results established we can now prove the following lemma.

Lemma A4. For $k=0(1) r, \tau \frac{1}{\tau} t$ then

$$
\frac{\partial^{k}}{\partial t^{k}}\left(\frac{g(\tau)-g(t)}{\tau-t}\right)=\frac{g_{k}(\tau, t)-g_{k}(t, t)}{\tau-t} .
$$

Proof. Proceed by induction. The result is obviously true for $k=0$; suppose it is true for $k=\ell$. Then

$$
\begin{aligned}
\frac{\partial^{\ell+1}}{\partial t^{\ell+1}}\left(\frac{g(\tau)-g(t)}{\tau-t}\right) & =\frac{\partial}{\partial t}\left(\frac{g_{\ell}(\tau, t)-g_{\ell}(t, t)}{\tau-t}\right) \\
& =\frac{1}{\tau-t}\left\{\left(\frac{\partial g_{\ell}}{\partial t}-g^{(\ell+1)}(t)\right)+\frac{g_{\ell}(\tau, t)-g^{(\ell)}(t)}{\tau-t}\right\} \text { by Lemma A3(i) } \\
& =\frac{1}{\tau-t}\left\{\frac{\ell}{\ell+1} g_{\ell+1}(\tau, t)-g^{(\ell+1)}(t)+\frac{1}{\ell+1} g_{\ell+1}(\tau, t)\right\}, \\
& =\frac{g_{\ell+1}(\tau, t)-g_{\ell+1}(t, t)}{\tau-t}, \quad \text { on Lemma A3(ii) and (iii) }
\end{aligned}
$$

The result now follows.

We now give a generalization of Theorem A2.

TheOREM A5. Suppose $g^{(r)} \in H_{\nu}[-1,1], 1 / q<\nu \leq 1$, where $w \in H\left(q_{1}, q_{2}\right)$, $1<q_{1}, q_{2}<\infty$ and $q=\min \left\{q_{1}, q_{2}\right\}$. If

$$
G(t)=\int_{-1}^{1} w(\tau)\left(\frac{g(\tau)-g(t)}{\tau-t}\right) d \tau, \quad t \in[-1,1]
$$

then $G^{(r)} \in H_{\nu-1 / q}[-1,1]$.

Proof. From Lemma A4 we can write

$$
G^{(r)}(t)=\int_{-1}^{1} w(\tau)\left(\frac{g_{r}(\tau, t)-g_{r}(t, t)}{\tau-t}\right) d \tau .
$$


When $r=0$, the result is that given by Theorem A1. When $r \geq 1$ we have, by Lemma A3(iv), that $g_{r} \in H_{\nu}[-1,1] \times H_{\nu}[-1,1]$. Following the proof of Theorem A1 we shall assume $h>0$ and let $t_{1}=t_{0}+h$. Again, we have three cases depending upon whether or not $t_{0}$ or $t_{1}$ is an end point of $[-1,1]$. Here we shall consider only case (i) where $\ell=\left[t_{0}-2 h, t_{0}+2 h\right]$ is in $(-1,1)$. The other two cases follow by similar arguments. Now

$$
\begin{aligned}
& G^{(r)}\left(t_{1}\right)-G^{(r)}\left(t_{0}\right) \\
& =\left[\int_{L}+\int_{\ell}\right]\left[w(\tau)\left\{\frac{g_{r}\left(\tau, t_{1}\right)-g_{r}\left(t_{1}, t_{1}\right)}{\tau-t_{1}}-\frac{g_{r}\left(\tau, t_{0}\right)-g_{r}\left(t_{0}, t_{0}\right)}{\tau-t_{0}}\right\}\right] d \tau=K_{1}+K_{2} .
\end{aligned}
$$

Consider first the integral $K_{2}$ over $\ell$. Since

$$
\left|g_{r}\left(\tau_{1}, t_{1}\right)-g_{r}\left(\tau_{2}, t_{2}\right)\right| \leq A_{r}\left\{\left|\tau_{1}-\tau_{2}\right|^{\nu}+\left|t_{1}-t_{2}\right|^{\nu}\right\}
$$

for all $\left(\tau_{1}, t_{1}\right),\left(\tau_{2}, t_{2}\right) \in[-1,1] \times[-1,1]$ we have that

$$
\left|K_{2}\right| \leq A_{r} \int_{\ell} w(\tau)\left\{\left|\tau-t_{1}\right|^{\nu-1}+\left|\tau-t_{0}\right|^{\nu-1}\right\} d \tau
$$

Using the same arguments as we did in the proof of Theorem A1 when estimating $I_{2}$ we find that

$$
\left|K_{2}\right| \leq \mathrm{ch}^{\nu-1 / q} \text {. }
$$

Consider now $K_{1}$, the integral over $L$. We can rewrite this as

$$
\begin{aligned}
K_{1} & =\left(g_{r}\left(t_{0}, t_{0}\right)-g_{r}\left(t_{1}, t_{1}\right)\right) \int_{L} \frac{w(\tau) d \tau}{\tau-t_{0}}+\int_{L} w(\tau)\left(\frac{g_{r}\left(\tau, t_{1}\right)-g_{r}\left(\tau, t_{0}\right)}{\tau-t_{0}}\right) d \tau \\
& +h \int_{L} w(\tau)\left(\frac{g_{r}\left(\tau, t_{1}\right)-g_{r}\left(t_{1}, t_{1}\right)}{\left(\tau-t_{0}\right)\left(\tau-t_{1}\right)}\right) d \tau .
\end{aligned}
$$

From the Hölder condition on $g_{r}$ we find

$$
\left|K_{1}\right| \leq 3 A_{r} h^{\nu} \int_{L} \frac{w(\tau) d \tau}{\left|\tau-t_{0}\right|}+A_{r} h \int_{L} \frac{w(\tau)\left|\tau-t_{1}\right|^{\nu-1}}{\left|\tau-t_{0}\right|} d \tau .
$$

Using the same techniques as we did for estimating the integrals $J_{3}$ and $J_{4}$ in Theorem A1 we find that $\left|K_{1}\right| \leq c h^{\nu-1 / q}$ so we have, in case (i), that

$$
\left|G^{(r)}\left(t_{1}\right)-G^{(r)}\left(t_{0}\right)\right| \leq c h^{\nu-1 / q} .
$$

A similar argument holds for cases (ii) and (iii) so that the theorem is proved.

We now give a generalization of Theorem A2. 
Theorem A6. Suppose $w \in H\left(q_{1}, q_{2}\right), 1<q_{1}, q_{2}<\infty$ and $q=\min \left\{q_{1}, q_{2}\right\}$. Suppose also that for some non-negative integer $r, g^{(r)} \in H_{\nu}[-1,1]$, where $1 / q<\nu \leq 1$. Then if $G=\hat{A}^{I} g$ we have that $G^{(r)} \in H_{\nu-1 / q}[-1,1]$.

Proof. If in equation (A7) we apply Theorem A5 to the appropriate terms on the right hand side, we see that $\hat{A}^{I} g$ is the sum of two terms each of which possesses a Hölder continuous derivative of order $r$.

\section{REFERENCES}

1. C.W Clenshaw, A note on the summation of Chebyshev series, M.T.A.C. 9 (1955), 118-120.

2. D. Elliott, Singular integral equations on the arc $(-1,1)$ : theory and approximate solution Part 1: theory, University of Tasmania, Math. Dept., Tech Rpt. No. 218 (1987)

3. M.A. Golberg, The numerical solution of Cauchy singular integral equations with constant coefficients, J. Int. Equns. 9 (1985), 127-151.

4. V. Hutson and J.S. Pym, Applications of functional analysis and operator theory, Academic Press, London, 1980.

5. A.I. Kalandiya, On a direct method of solution of an equation in wing theory and its application to the theory of elasticity, Mat. Sb. 42 (1957), 249-272.

6. B.V. Khevedelidze, Linear discontinuous boundary problems in the theory of functions, singular integral equations and some of their applications, Akad. Nauk. Gruzin S.S.R. 23 (1956), 3-158.

7. N.I. Muskhelishvili, Singular integral equations, Noordhoff, Groningen, 1953.

8. T.J. Rivlin, An introduction to the approximation of functions, Blaisdell, Waltham, 1969.

9. The Chebyshev polynomials, John Wiley, New York, 1974.

10. E. Venturino, Recent developments in the numerical solution of singular integral equations, J. Math. Anal. Appln. 115 (1986), 239-277.

Department of Mathematics, University of TAsmania, Box 252C G.P.O., Hobart, Tasmania, 7001, Australia. 